\title{
AVALIAÇÃO DA FUNCIONALIDADE EM IDOSAS INSTITUCIONALIZADAS
}

\author{
Evaluation of functionality in elderly institutionalized \\ Evaluación de la funcionalidad en las edades institucionalizadas
}

\section{Joselito de Oliveira Neto ${ }^{* 1}$, Monik Batista Ferreira da Costa ${ }^{2}$, Naégina}

Fernandes de Almeida ${ }^{2}$, Raquel Horn Vasconcelos de Oliveira ${ }^{3}$ e Daniela Gardano Bucharles Mont'Alverne ${ }^{4}$

${ }^{I}$ Núcleo de Pesquisa e Desenvolvimento de Medicamentos (NPDM), Fisioterapeuta e Doutorando em

Biotecnologia, Universidade Federal do Ceará (UFC), Fortaleza - Ce, Brasil.

${ }^{2}$ Laboratório de Fisioterapia, Curso de Fisioterapia, UniNassau, Fortaleza-Ce, Brasil.

${ }^{3}$ Centro de Ciências da Saúde, Universidade de Fortaleza (Unifor), Fortaleza-Ce, Brasil.

${ }^{4}$ Departamento de Fisioterapia, Docente Adjunta da Universidade Federal do Ceará (UFC), Fortaleza-Ce,

Brasil.

*Correspondência: Núcleo de Pesquisa e Desenvolvimento de Medicamentos (NPDM), Universidade Federal do Ceará - UFC, Rua Coronel Nunes de Melo, 1000 - Rodolfo Teófilo, Fortaleza - CE Brasil. e-mail joselitoneto@yahoo.com.br

\section{RESUMO}

Com o envelhecimento o corpo humano apresenta diversas modificações, podendo alterar a capacidade funcional do idoso, tendo em vista o aparecimento de doenças crônicas e podendo afetar as AVD'S desses idosos. O presente estudo teve como objetivo avaliar a funcionalidade das idosas institucionalizadas em casas de repouso na cidade de Fortaleza, Ceará, Brasil. Trata-se de um estudo de campo do tipo observacional com caráter quantitativo, cuja pesquisa foi realizada em duas Instituições de Longa Permanência para Idosos (ILPI's). Participaram 25 idosas residentes nos locais de estudo, cujo os dados foram coletados por meio do Índice de Katz. Os dados foram apresentados por análise descritiva. A Independência foi observada em 64\% (n=16), a Dependência Parcial em 12\% ( $\mathrm{n}=3)$ e a Dependência Importante em 24\% ( $\mathrm{n}=6)$, sendo que as idosas apresentam melhor capacidade de execução das atividades de continência $(84 \% ; n=21)$ e banho $(80 \% ; n=20)$. Assim, o presente estudo mostra a necessidade de acompanhamento dessas idosas para execução de suas AVD's com o aumento da idade.

Palavras-chave: Avaliação funcional. Envelhecimento. Instituição de Longa Permanência.

\section{ABSTRACT}

With the aging the human body presents several modifications, being able to alter the functional capacity of the elderly, in view of the appearance of chronic diseases and can affect the ADLs of these elderly people. The present study will evaluate the functionality of institutionalized elderly women in nursing homes in the city of Fortaleza, Ceará, Brazil. This is an observational field study with a quantitative character, whose research was carried out in two Long-Term Institutions for the Elderly (LTIE's). Participants were 25 elderly women living in the study sites, whose data were collected through the Katz Index. Data were presented by descriptive analysis. Independence was observed in 64\% ( $\mathrm{n}=16)$, Partial Dependence in $12 \%(\mathrm{n}=3)$ and Important Dependence in $24 \%(\mathrm{n}=6)$, and the elderly presented a better capacity to perform continence activities $(84 \%, \mathrm{n}=21)$ and bath $(80 \%, \mathrm{n}=20)$. Thus, the present study shows the need for follow-up of these elderly women to perform their ADLs with increasing age.

Keywords: Functional evaluation. Aging. Institution of Long Stay. 


\section{RESUMEN}

Con el envejecimiento el cuerpo humano presenta diversas modificaciones, pudiendo alterar la capacidad funcional del anciano, teniendo en vista la aparición de enfermedades crónicas y pudiendo afectar a las AVD'S de esos ancianos. El presente estudio tuvo como objetivo evaluar la funcionalidad de las ancianas institucionalizadas en casas de reposo en la ciudad de Fortaleza, Ceará, Brasil. Se trata de un estudio de campo del tipo observacional con carácter cuantitativo, cuya investigación fue realizada en dos Instituciones de Larga Permanencia para ancianos (ILPI's). Participaron 25 ancianos residentes en los lugares de estudio, cuyos datos fueron recolectados a través del Índice de Katz. Los datos fueron presentados por análisis descriptivo. La Independencia fue observada en el 64\% (n = 16), la Dependencia Parcial en el $12 \%(\mathrm{n}=3)$ y la Dependencia Importante en el $24 \%(\mathrm{n}=6)$, siendo que las ancianas presentan mejor capacidad de ejecución de las actividades de continencia $(84 \%, \mathrm{n}=21)$ y baño $(80 \%, \mathrm{n}=$ 20). Así, el presente estudio muestra la necesidad de acompañamiento de esas ancianas para la ejecución de sus AVD's con el aumento de la edad.

Descriptores: Evaluación funcional. Envejecimiento. Institución de larga permanencia.

\section{INTRODUÇÃO}

O envelhecimento é um processo natural da vida que promove alterações biológicas, psicológicas e sociais no indivíduo. Todas essas transformações variam de acordo com as características genéticas, fatores externos, como modo de vida e hábitos de cada pessoa (SOUZA; SERRA; SUZUKI, 2012). As consequências geradas pelo envelhecimento podem levar a um maior risco de transtornos e doenças. A prevalência de doenças crônicas contribui para a redução da capacidade física e biológica, portanto, reduz a independência e autonomia (VICENTIN et al., 2015).

O conjunto das alterações fisiológicas e patológicas vivenciadas pelos idosos culmina com a crescente dependência, que consiste em um processo dinâmico, cuja evolução pode se modificar e até ser prevenida ou reduzida, se houver ambiente e assistência adequados (VERAS, 2009).

Com essa crescente proporção de idosos e longevidade, juntamente com as dificuldades sócio econômicas, diferenças culturais, conflitos familiares que envolvem os idosos e seus cuidadores, o declínio da saúde dos senescentes, fazem crescer a demanda por Instituições de Longa Permanência para Idosos (ILPI's), definidas pela portaria da Secretaria de
Estado da Assistência Social (SEAS) no 2874/2000 (BRASIL, 2000).

A Sociedade Brasileira de Geriatria e Gerontologia (SBGG) sugeriu em 2003 a adoção da denominação de ILPI, pois se notou a redução das capacidades funcionais dos idosos e que eles necessitavam não apenas de um abrigo, mas da assistência à saúde em geral (SBGG, 2003).

As ILPI's são modalidades de caráter residencial, que recebem pessoas com idade igual ou superior a 60 anos, com ou sem suporte familiar, em condição de liberdade, dignidade e cidadania, podendo ou não estar vinculadas à iniciativa governamental (AIRES, 2009; TAVARES, 2007). Essas Instituições tem sua origem relacionada com os asilos, que tinham a função de acolher pessoas com a necessidade de abrigo e da caridade cristã, em meio à precariedade de políticas públicas que desempenhassem essas funções (CAMARO \& KANSO, 2010).

Os idosos apresentam características individuais, características estas que tem relação com sua história de vida, com seu grau de independência funcional e com a demanda por serviços mais ou menos específicos, por este motivo podemos dizer que eles são diferentes. Devido a este ponto de vista, 
os idosos precisam de uma avaliação genérica e adaptada na realidade cultural em que eles estão inseridos (FARIAS, 2007).

Avaliação funcional é definida como uma tentativa sistematizada de medir, de forma objetiva, os níveis nos quais uma pessoa é capaz de desempenhar determinadas atividades ou funções, utilizando-se de algumas habilidades para o desempenho das tarefas da vida cotidiana. Assim, cresce, também, a necessidade de utilização de instrumentos de avaliação funcional. O Índice de Independência nas Atividades de Vida Diária (AVD's), desenvolvido por Sidney Katz, é um dos instrumentos mais antigos e também dos mais citados na literatura nacional e internacional (DUARTE; ANDRADE; LEBRÃO, 2007).

A dependência pode ser considerada, ainda, como um estado em que as pessoas se encontram por razões ligadas à falta ou perda de autonomia (física, psíquica, social), de necessidade de ajuda para realizar as AVD's. É um problema grave de saúde que interfere na qualidade de vida do idoso e do seu cuidador (ARAÚJO \& CEOLIM, 2007).

Considera-se que a institucionalização do idoso pode resultar em declínio funcional, com consequente perda da independência para o desempenho das AVD's. Nesse sentido, justifica-se a importância de realizar estudos acerca do grau de dependência dos idosos residentes nas ILPI's, uma vez que esses resultados podem contribuir para subsidiar ações que proporcionem melhor qualidade de vida a esses idosos (ARAÚJO, 2007; CREUTZBERG, 2008).

O processo de envelhecimento caracteriza-se para muitos idosos, a perda de autonomia e independência, sendo as ILPI's cada vez mais procuradas por familiares na tentativa de mantê-los institucionalizados (PEREIRA et al., 2004).
Neste contexto, este trabalho teve como objetivo avaliar a funcionalidade de idosas institucionalizadas na cidade de Fortaleza - CE.

\section{MATERIAIS E MÉTODOS}

Trata-se de um estudo de campo do tipo descritivo observacional com caráter quantitativo, desenvolvido em duas ILPI's localizadas na cidade de Fortaleza - CE, no período de março a abril de 2017.

Participaram da pesquisa 25 idosas (idade igual ou superior a 60 anos completos) residentes nas Instituições em estudo. Para fins de coleta de dados, foram excluídas idosas com déficit cognitivo severo e aquelas idosas com dificuldades para responder às perguntas durante a abordagem pelo entrevistador (a), foram auxiliados nas respostas por um cuidador.

Os dados foram coletados através de uma entrevista realizada com as idosas individualmente. Tivemos como instrumento de coleta o Índice de Katz, que se trata de uma escala com diferentes graus de independência funcional, nos atos de banhar-se, vestir-se, ir ao banheiro, transferência, continência e alimentação. Cada atividade recebe uma pontuação que varia de independente, parcialmente dependente ou totalmente dependente. Ao total de pontos é relatado 6 como independente, 4 como dependência moderada e abaixo de 2 muito dependente. De acordo com Katz et al. (1963), este formulário foi desenvolvido para facilitara avaliação dos observadores e o registro mais preciso das informações, como mostra a Figura 1. As variáveis foram extraídas mediante os resultados e respostas encontradas.

As idosas foram convidadas e receberam orientações sobre o estudo e aquelas que concordaram em participar assinaram o Termo de Consentimento Livre e Esclarecido - TCLE conforme preconiza a Resolução $N^{\circ}$ 196/196 do Conselho Nacional de Saúde. A pesquisa segura os aspectos 
éticos que envolvem a pesquisa com seres humanos, como garantia de confiabilidade do anonimato, da não utilização das informações somente para fins previstos na pesquisa.

\section{Figura1: Índice de Katz.}

\begin{tabular}{|c|c|c|c|c|c|}
\hline $\begin{array}{l}\text { ATIVIDADES } \\
\text { Pontos (1 ou } 0)\end{array}$ & \multicolumn{3}{|c|}{$\begin{array}{l}\text { PENDENCIA } \\
\text { (1 ponto) } \\
\text { entaçáo ou assiténcia pessoal }\end{array}$} & \multicolumn{2}{|c|}{$\begin{array}{c}\text { DEPENDENCIA } \\
\text { (0 pontos) } \\
\text { COM supervisáo, orientaçao ou assitência pessoal } \\
\text { ou cuidado integral }\end{array}$} \\
\hline $\begin{array}{l}\text { nhar-se } \\
\text { Pontos: }\end{array}$ & \multicolumn{3}{|c|}{$\begin{array}{l}\text { (1 ponto) Banha-se completamente ou necessita de } \\
\text { auxilio somente para lavar uma parte do corpo como } \\
\text { as costas, genitais ou uma extremidade incapacitada }\end{array}$} & \multicolumn{2}{|c|}{$\begin{array}{l}\text { (0 pontos) Necessita de ajuda para banhar-se em } \\
\text { mais de uma parte do corpo, entrar e sair do chuveiro } \\
\text { ou banheira ou requer assistencia total no banho }\end{array}$} \\
\hline $\begin{array}{l}\text { Stir-se } \\
\text { Pontos: }\end{array}$ & \multicolumn{3}{|c|}{$\begin{array}{l}\text { (1 ponto) Pega as roupas do armário e veste as roupas } \\
\text { intimas, externas e cintos. Pode receber ajuda para } \\
\text { amarrar os sapatos }\end{array}$} & \multicolumn{2}{|c|}{$\begin{array}{l}\text { (0 pontos) Necessita de ajuda para vestir-se ou } \\
\text { necessita ser completamente vestido }\end{array}$} \\
\hline Pontos: & \multicolumn{3}{|c|}{$\begin{array}{l}\text { (1 ponto) Dirigi-se ao banheiro, entra e sai do mesmo, } \\
\text { arruma suas proprias roupas, limpa a área genital } \\
\text { sem ajuda }\end{array}$} & \multicolumn{2}{|c|}{$\begin{array}{l}\text { (0 pontos) Necessita de } \\
\text { limpar-se ou usa urinol }\end{array}$} \\
\hline Pontos: & \multicolumn{3}{|c|}{$\begin{array}{l}\text { (1 ponto) Senta-seldeita-se e levanta-se da cama ou } \\
\text { cadeira sem ajuda. Equipamentos mecánicos de ajuda } \\
\text { sâo aceitaveis }\end{array}$} & \multicolumn{2}{|r|}{$\begin{array}{l}\text { aara sentar-se/deitar- } \\
\text { deira }\end{array}$} \\
\hline $\begin{array}{l}\text { ntinência } \\
\text { Pontos: }\end{array}$ & \multicolumn{3}{|c|}{$\begin{array}{l}\text { (1 ponto) Tem completo controle sobre suas eliminaçes } \\
\text { (urinar e evacuar) }\end{array}$} & \multicolumn{2}{|c|}{$\begin{array}{l}\text { (0 pontos) E parcial ou totalmente incontinente do } \\
\text { intestino ou bexiga }\end{array}$} \\
\hline $\begin{array}{l}\text { mentaçáo } \\
\text { Pontos: }\end{array}$ & \multicolumn{3}{|c|}{$\begin{array}{l}\text { (1 ponto) Leva a comida do prato a boca sem ajuda. } \\
\text { Preparaçå da comida pode ser felta por outra pessoa }\end{array}$} & \multicolumn{2}{|c|}{$\begin{array}{l}\text { (0 pontos) Necessita de ajuda parcial ou total com a a } \\
\text { alimentaçáo ou requer alimentaçâo parenteral }\end{array}$} \\
\hline$=$ & & $0=$ & & tra & 2 ou \\
\hline
\end{tabular}

Fonte: JUNIOR \& RAISER,2016.

A análise de dados foi realizada no software Microsoft Excel 2016. Os valores são mostrados em termos de números e percentuais. Os resultados são mostrados em termos do grau de dependência e da capacidade de execução de atividades entre os pacientes entrevistados.

A pesquisa seguiu a todos os procedimentos éticos de pesquisa e cumpriu as "Diretrizes e Normas Regulamentadoras de Pesquisa Envolvendo Seres Humanos" (466/12) editadas pela Comissão Nacional de Ética e Pesquisa (CONEP) sendo aprovado com o parecer número 347\2011.

\section{RESULTADOS E DISCUSSÃO}

O estudo foi conduzido com 25 idosas em duas Instituições. Na Instituição A, participaram sete idosas e dezoito na Instituição B. A Independência foi observada em $64 \% \quad(n=16)$, a Dependência Parcial em 12\% $(n=3)$ e a Dependência Importante em 24\% $(n=6)$. Os resultados apontam para o aumento da ocorrência de Grau de Dependência em especial, Dependência Importante com o aumento da idade (Tabela 01).
$\mathrm{Na}$ Tabela 2, encontra-se o grau de dependência das idosas institucionalizadas em relação às seis Atividades de Vida Diárias (AVD'S), relativas ao autocuidado. Os resultados mostraram que as idosas apresentaram melhor capacidade de execução das atividades de continência $(84 \% ; n=21)$ e banho $(80 \% ; n=20)$. Por outro lado, as atividades de transferência $(72 \% ; n=18)$ e higiene pessoal $(72 \%$; $\mathrm{n}=18$ ) foram aquelas em que tiveram menor capacidade de execução.

Tabela 1. Distribuição das idosas, segundo Grau de Dependência (Índice de Katz) e faixa etária, Fortaleza, CE.

\begin{tabular}{|c|c|c|c|c|c|c|c|c|}
\hline \multirow{2}{*}{ Faixa Etária } & \multicolumn{2}{|c|}{ Independência } & \multicolumn{2}{|c|}{ Dependência Parcial } & \multicolumn{2}{|c|}{ Dependência Importante } & \multicolumn{2}{|c|}{ Total } \\
\hline & $\mathrm{n}$ & $\%$ & $\mathrm{n}$ & $\%$ & $\mathrm{n}$ & $\%$ & $\mathrm{n}$ & $\%$ \\
\hline $60-69$ & 5 & 20 & e & $\ell$ & $\ell$ & $\ell$ & 2 & 20 \\
\hline $70-79$ & 5 & 20 & 1 & 4 & $\ell$ & $\ell$ & 6 & 24 \\
\hline $80-89$ & 6 & 24 & $\ell$ & $\ell$ & 1 & 4 & 2 & 28 \\
\hline $90-99$ & $\ell$ & $\ell$ & 2 & $\&$ & ई & 20 & 2 & 28 \\
\hline Total & 16 & 64 & 3 & 12 & 6 & 24 & 25 & 100 \\
\hline
\end{tabular}

Fonte: Dados da Pesquisa.

Tabela 2. Distribuição das idosas segundo a capacidade de execução das AVD’s, Fortaleza, CE.

\begin{tabular}{l|ll||cc}
\hline \hline \multirow{2}{*}{ Itens } & \multicolumn{2}{|c||}{ Independente } & \multicolumn{2}{c}{ Dependente } \\
\cline { 2 - 5 } & $\mathbf{n}$ & $\%$ & $\mathbf{n}$ & $\%$ \\
\hline \hline Banho & 20 & 80 & 5 & 20 \\
\hline Vestir-se & 19 & 76 & 6 & 24 \\
\hline Higiene Pessoal & 18 & 72 & 7 & 28 \\
\hline Transferência & 18 & 72 & 7 & 28 \\
\hline Alimentação & 19 & 76 & 6 & 24 \\
\hline Continência & 21 & 84 & 4 & 16 \\
\hline \hline
\end{tabular}

Fonte: Dados da pesquisa.

O presente estudo permitiu a descrição em relação ao grau de dependência das idosas institucionalizadas. A maioria apresentou-se com grau de independência na realização de AVD's.

Esta pesquisa mostrou que $32 \%$ das idosas acima de 80 anos apresentam algum grau de dependência. Esses resultados não diferem daqueles encontrados em outras pesquisas envolvendo o mesmo grupo populacional (MARINHO et al., 2013). Em Montes Claros, 125 idosos de uma Instituição de Longa Permanência foram avaliados por meio do Índice de Katz e a metade desses idosos possuía 
algum grau de dependência (MARINHO et al., 2013). Em outra investigação, verificou-se que, no grupo etário dos 80 aos 90 anos, para as AVD's, 69\% dos idosos eram dependentes e no grupo entre 70 e 79 anos, $14 \%$ o eram (ROSA et al., 2003).

Em estudo realizado por Pelegrin e colaboradores (2008) mostrou que, as atividades em que os idosos apresentaram maior dependência, foram tomar banho e vestir-se, diferentemente desse estudo, onde foi encontrado maior dependência para as atividades de higiene pessoal e transferência.

A predominância do sexo feminino nesse estudo é possivelmente justificada pela atual feminização da velhice, decorrente da maior expectativa de vida da mulher no Brasil (AIRES; PAZ; PEROSA, 2009).

Em relação ao grau de dependência comparado a grupos etários, observou-se o aumento no percentual de idosos com dependência importante com o avançar da idade, com diferença estatisticamente significativa entre os grupos. Estudo desenvolvido em Instituições de Longa Permanência, em 2005, revelou progressão no declínio cognitivo e funcional, à medida que os idosos envelhecem (ARAÚJO \& CEOLIM, 2007).

O processo de envelhecimento traz consigo uma redução na qualidade e quantidade das informações necessárias para um controle motor e cognitivo eficaz e alguns sistemas orgânicos experimentam esse declínio, tendendo a ser linear em decorrência do tempo, sem definir um ponto exato de transição, como ocorre nas outras fases da vida (MACIEL \& GUERRA, 2007).

É condição fundamental para a manutenção de sua independência física, comportamental e cognitiva estimular a independência do idoso institucionalizado em qualquer grupo etário (MARINHO et al., 2013).
A escassez de estímulos que as Instituições proporcionam ao idoso causa redução do potencial para autonomia e interfere na qualidade do envelhecer. Alterações da cognição podem estar relacionadas às alterações na execução das atividades que determinam a capacidade funcional $\mathrm{e}$ a institucionalização de um idoso pode influenciar o comprometimento de suas funções autônomas. (GREVE et al., 2007).

Os determinantes da incapacidade funcional são multifatoriais. A presença dos mesmos fatores de risco para a limitação funcional em indivíduos diferentes pode gerar manifestações diversas, com diferentes repercussões nas AVD's. O ambiente social e físico e os fatores emocionais, econômicos e de saúde interagem na expressão de todo o potencial funcional do idoso (DANILOW et al., 2007).

A dependência não é um estado permanente, mas um processo dinâmico, cuja evolução pode ser modificada, prevenida e/ou reduzida. Tal evolução nesse processo sugere a existência de serviços qualificados e comprometidos com a assistência ao idoso. As necessidades de cuidado requerem desenvolvimento das AVD's, à medida que, impossibilitado para o autocuidado, a instituição assume o papel de provedor desses cuidados (ARAUJO \& CEOLIM, 2007).

\section{CONCLUSÃO}

Diante dos resultados obtidos neste presente trabalho, a maioria das idosas é independente nas suas AVD's.

As idosas que apresentam algum grau de dependência para o desempenho das AVD's constituem a minoria nas ILPI's, e apresentam dificuldades para transferência e higiene pessoal, assim, necessitando de um maior acompanhamento.

Nesse sentido, o envelhecimento populacional vem trazendo desafios para a sociedade, 
exigindo uma melhor adaptação nas Instituições, para assegurar atenção integral à saúde e melhor qualidade de vida aos idosos institucionalizados.

\section{AGRADECIMENTO}

À direção das Instituições de Longa Permanência de Idosos que autorização a realização da pesquisa.

Todos os autores declararam não haver qualquer potencial conflito de interesses referente a este artigo.

\section{REFERÊNCIAS}

AIRES, M.; PAZ A. A.; PEROSA C.T. Situação de saúde e grau de dependência de pessoas idosas institucionalizadas.

Revista

Gaúcha de Enfermagem. v. 30, n. 3, p. 492-9, 2009.

ARAÚJO, M; CEOLIM, M.F. Avaliação do graude independência de idosos residentes em instituições de longa permanência. Revista da Escola de Enfermagem da USP. v. 41, n. 3, p. 378-85, 2007.

BRASIL. Secretaria de Ação Social SEAS. Portaria $n^{\circ}$ 2874/2000. Instituições de Longa Permanência para Idosos (ILPI's). Diário Oficial da União. 20 jul. 2000.

CAMARANO, A.A; KANSO, S. As instituições de longa permanência para idosos no Brasil. Revista brasileira de estudos de população, v. 27 , n. 1, p. 232-235, 2010.

CREUTZBERG, M; GONÇALVES, L.H.T; SOBOTTKA E.A. Instituição de longa permanência para idosos: a imagem que permanece. Texto \& Contexto Enfermagem, v. 17, n. 2, p. 273-9, 2008.

DANILOW, M.Z, MOREIRA, A.C.S; VILELA, C.G; BARRA, B.B; NOVAES, M.R.C.G, OLIVEIRA, M.P.F. Perfil epidemiológico, sociodemográfico e psicossocial de idosos institucionalizados do Distrito Federal. Comunicação em ciências da saúde, v. 18, n.1, p. 9-16, 2007.

DUARTE, Y.A.O; ANDRADE, C.L; LEBRÃO, M.L; O Índex de Katz na avaliação da funcionalidade dos idosos. Revista da Escola de Enfermagem, v. 41, n. 2, p. 317-25, 2007.
FARIAS, C.E.S; Avaliação funcional do idoso. Monografia de Conclusão de Curso, Curso de Fisioterapia. Universidade Veiga de Almeida. Rio de Janeiro, 2007.

GREVE, P; GUERRA, A.G, PORTELA, M.A; PORTES, M.S, REBELATTO; J.R. Correlações entre mobilidade e independência funcional em idosos institucionalizados e não institucionalizados. Revista Fisioterapia em Movimento, v. 20, n. 4, p. 117- 24, 2007.

JUNIOR, A.A.P; RAISER, G.M. Avaliação do grau de independência funcional de idosos institucionalizados por meio do Índice de Katz da cidade de Blumenau. Revista Maiêutica, Indaial, v. 3, n. 1, p. 43-52, 2016.

KATZ S. et al. Studies of illness in the aged. The index of ADL: a standardized measure of biological and psychosicial function. JAMA. v. 185, n. 12, 1963.

MACIEL, A.C.C; GUERRA, R.O. Influência dos fatores biopsicossociais sobre a capacidade funcional de idosos residentes no Nordeste do Brasil. Revista Brasileira de Epidemiologia, São Paulo, v. 10, n. 2, p. 178-189, 2007.

MARINHO, L.M.; VIEIRA, M.A; COSTA, S.M; ANDRADE, J.M.O. Grau de dependência de idosos residentes em instituições de longa permanência. Revista Gaúcha de Enfermagem, v.34, n.1, Mar. 2013.

PELEGRIN, A. K. A. P. ARAÚJO, J. A.; COSTA, L. C.; CYRILLO, R. M. Z.; ROSSET, I. Idosos de Uma Instituição de Longa Permanência de Ribeirão Preto: Níveis de Capacidade Funcional. Revista Arquivos de Ciências da Saúde, v.15, n.4, p.182- 8, 2008.

PEREIRA, L.S.M; BRITTO, R.R; VALADARES, M.C; PEREIRA, E.F.S. Programa de melhoria da qualidade de vida dos idosos institucionalizados; 2004.

ROSA, T.E; BENICIO, M.H; LATORRE, M.R; RAMOS, L.R. Fatores determinantes da capacidade funcional entre idosos. Revista de Saúde Pública, v. 37, n.1, p. 40-8, 2003.

SBGG - Sociedade Brasileira de Geriatria e Gerontologia - Seção São Paulo. Instituição de Longa Permanência para Idosos: Manual de 
Funcionamento. Sociedade Brasileira de Geriatria e Gerontologia - Seção São Paulo, p. 39, 2003.

SOUZA, D.B; SERRA, A.J; SUZUKI, F.S; Atividade física e nível de depressão em idosos. Revista Brasileira de Ciências da Saúde, v. 16, n. 1, p. 2-6, 2012.

TAVARES, D.M.S; PEREIRA, G.A; IWAMOTO, H.H; MIRANZZI, S.S.C; RODRIGUES, L.R; MACAHADO, A.R.M. Incapacidade funcional entre idosos residentes em um município do interior de Minas Gerais. Texto \& Contexto Enfermagem, v.16, n.1, p. 32-9, 2007.
VERAS, R. Envelhecimento populacional contemporâneo: demandas, desafios e inovações. Rio de Janeiro. Revista de Saúde Pública, v. 43, n.3, p. 548-554, 2009.

VISENTIN, A; MANTOVANI, M.F; CAVEIÃO, C; MENDES, T.A; NEVES, A.S; HEY, A.P. Qualidade de vida de idosa hipertensa de uma instituição de longa permanência. Revista da Rede de Enfermagem do Nordeste, v. 16, n. 2, p. 218-25, mar/abr. 2015. 